\title{
Stroke and food groups: an overview of systematic reviews and meta-analyses
}

\author{
Cuiyu Deng ${ }^{1} \dagger$, Qi Lu ${ }^{2} \uparrow$, Bingyan Gong ${ }^{2}$, Liya Li ${ }^{2}$, Lianxia Chang ${ }^{3}$, Li Fu ${ }^{1, *}$ \\ and Yue Zhao ${ }^{2, *}$ \\ ${ }^{1}$ Nursing Department, The Second Hospital of Tianjin Medical University, Pingiiang Road, Hexi District, Tianjin \\ 300211 , People's Republic of China: ${ }^{2}$ School of Nursing, Tianjin Medical University, Observatory Road, Heping \\ District, Tianjin 300070, People's Republic of China: ${ }^{3}$ Nursing Department, Tianjin First Center Hospital, Tianjin, \\ People's Republic of China
}

Submitted 2 August 2016: Final revision received 3 September 2017: Accepted 11 September 2017: First published online 16 November 2017

\begin{abstract}
Objective: Numerous systematic reviews of prospective studies on the association of stroke risk with the consumption of various food groups have been published. A review of the evidence across the existing systematic reviews and meta-analyses of prospective studies was conducted to provide an overview of the range and validity of the reported associations of food groups with stroke risk.

Design: The PubMed, EMBASE and Cochrane Library databases were searched for articles published up to September 2015 to identify systematic reviews of prospective studies.

Results: A total of eighteen studies published from 2008 to 2015 were eligible for analysis. Overall, thirteen specific foods were studied for an association with stroke outcome, including nuts, legumes, fruits and vegetables, refined grains, whole grains, dairy products, eggs, chocolate, red and/or processed meat, fish, tea, sugar-sweetened beverages and coffee. Whereas a high consumption of nuts, fruits, vegetables, dairy foods, fish and tea, and moderate consumption of coffee and chocolate demonstrated a protective effect, a high consumption of red and/or processed meat was associated with increased stroke risk. Refined grain, sugar-sweetened beverage, legume, egg and whole grain intake showed no effect on stroke outcome.

Conclusions: The current overview provided a high level of evidence to support the beneficial effect of specific foods on stroke outcome. Clinicians and policy makers could inform clinical practice and policy based on this overview.
\end{abstract}

Stroke is one of the leading causes of disabilities and death in both developed and developing countries. For example, stroke was the leading cause of death in China in 2010 (1.7 million deaths $)^{(1)}$. Due to population ageing, stroke, which consumes substantial health resources and leads to a significant economic burden, will become a tremendous burden to the health system in future decades ${ }^{(2)}$. However, stroke has a profound impact not only on the government but also on individuals and their families. Therefore, it is imperative to develop prevention strategies that might reduce the risk of stroke.

Diet is one of the key lifestyle factors involved in the prevention of stroke. Recently, extensive epidemiological studies have focused on the relationship among nutrients,

$\dagger$ Cuiyu Deng and Qi Lu contributed equally to this work. food groups, dietary patterns and stroke. For the general public, specific nutrition is too specialized to understand. Further, changing dietary patterns is also difficult for individuals. Compared with nutrients and dietary patterns, because food groups are more easily understood by and acceptable to the public, the targeted aim of the present overview was food groups. It has been shown that some specific foods are related to the risk of stroke, such as vegetables and fruits ${ }^{(3)}$, meat ${ }^{(4)}$ and fish ${ }^{(5)}$. Summarizing the evidence on the impact of diet at the food level will provide greater insights into the role of food groups in stroke.

An overview of systematic reviews is a relatively new approach to synthesizing a large body of literature in a particular area. Numerous systematic reviews of prospective studies regarding food groups associated with stroke have been published ${ }^{(6-8)}$. Systematic reviews of 
prospective studies are considered to provide a high level of evidence and represent a potentially powerful method to evaluate the long-term effects of food groups on stroke risk. Patients and researchers require such evidence to support shared decisions and to set priorities for research. Clinicians and policy makers also require information from systematic reviews to inform clinical practice and policy ${ }^{(9)}$. However, to date, there has been no effort to summarize the evidence from these systematic reviews and metaanalyses. As the first review of the evidence across existing systematic reviews of prospective studies, the present study provides an overview of the range and validity of the reported associations of food groups with stroke.

\section{Methods}

\section{Literature search}

The present systematic review was guided by the PRISMA Statement (Preferred Reporting Items for Systematic Reviews and Meta-Analyses $)^{(10)}$. A systematic literature search was conducted in the PubMed, EMBASE and Cochrane Library databases up to September 2015. Search terms included 'food', 'diet', 'nutrition', or one specific food such as 'nut', 'legume', 'fruit', 'vegetable', 'fish', 'meat', 'egg' and others, combined with 'stroke', 'cerebral vascular disease', 'cerebral infarction' and 'systematic review' and 'meta-analysis'. These searches included a mixture of MeSH (Medical Subject Headings) terms and text words to attempt to maximize the retrieval of potentially relevant studies (see online supplementary material for the search strategy and terms used). The reference lists of the retrieved articles were also reviewed.

\section{Study selection}

The eligibility criteria for including reviews in our overview were that the target review was itself a systematic review or meta-analysis of prospective studies and that it included studies investigating the relationship between a specific food or food groups and stroke outcome (including risk of stroke or stroke mortality). Studies in which CVD outcomes were assessed cumulatively rather than by individual disease risk assessment were not included. When more than one metaanalysis on the same research question was eligible and when the method of these systematic reviews or metaanalyses was similar, the study with the largest number of component studies with data on the individual studies' effect sizes was retained for the main analysis. When one systematic review or meta-analysis was an updated review of a previous review, we reported the results of the updated study only. Narrative, non-systematic reviews and editorials/commentaries were not eligible for this overview.

\section{Data extraction}

The following data were extracted from the selected studies using a standardized data-collection form: the name of the first author; publication year; targeted food; number of included studies; population size; case numbers; and conclusions of the meta-analysis. Two independent reviewers (C.D. and Q.L.) conducted the literature search, study selection and data extraction, removing duplicates or ineligible reviews before proceeding to full-text screening of the eligible articles. Consensus was reached through a third researcher's opinion (Y.Z.).

\section{Quality assessment}

The quality of the studies was assessed by two raters according to the AMSTAR (Assessment of Multiple Systematic Reviews) validated scale. The AMSTAR provides a risk of bias assessment across eleven different domains. The AMSTAR items are scored as 'yes', 'no', 'can't answer' or 'not applicable'. It has been shown to be adequately valid and reliable for use in assessing systematic reviews ${ }^{(11)}$. The quality of the studies was categorized as follows according to the number of criteria that were met: excellent, 11 ; very good, 9 or 10; good, 7 or 8; acceptable, 5 or 6; and deficient, 0 to 4 . The AMSTAR criteria include the following:

1. a priori design provided;

2. duplicate study selection/data extraction;

3. comprehensive literature search;

4. status of publication as inclusion criterion;

5. list of studies (included/excluded) provided;

6. characteristics of included studies documented;

7. scientific quality assessed and documented;

8. appropriate formulation of conclusions;

9. appropriate methods of combining studies;

10. assessment of publication bias; and

11. conflict of interest statement.

\section{Data synthesis}

The characteristics of the systematic reviews and statistical pooling of the included articles were summarized descriptively. Evidence tables were produced to synthesize the clinical findings and recommendations of the systematic reviews.

\section{Results}

\section{Description of the included studies}

The literature search identified 1027 relevant reviews. After duplicates were removed, 926 reviews remained for analysis (479 from PubMed, 401 from EMBASE and forty-six from the Cochrane Library). After screening of the abstracts, 108 reviews were retrieved as full text. In all, fifty-three reviews were identified as potential studies for inclusion in the overview. Ultimately, eighteen reviews fulfilled the inclusion criteria and were included in the overview, all of which were published between 2008 and 2015 (Fig. 1).

The characteristics and results of the included reviews are presented in Table 1. The reviews examined the following topics: nuts, legumes, fruits and vegetables, refined 


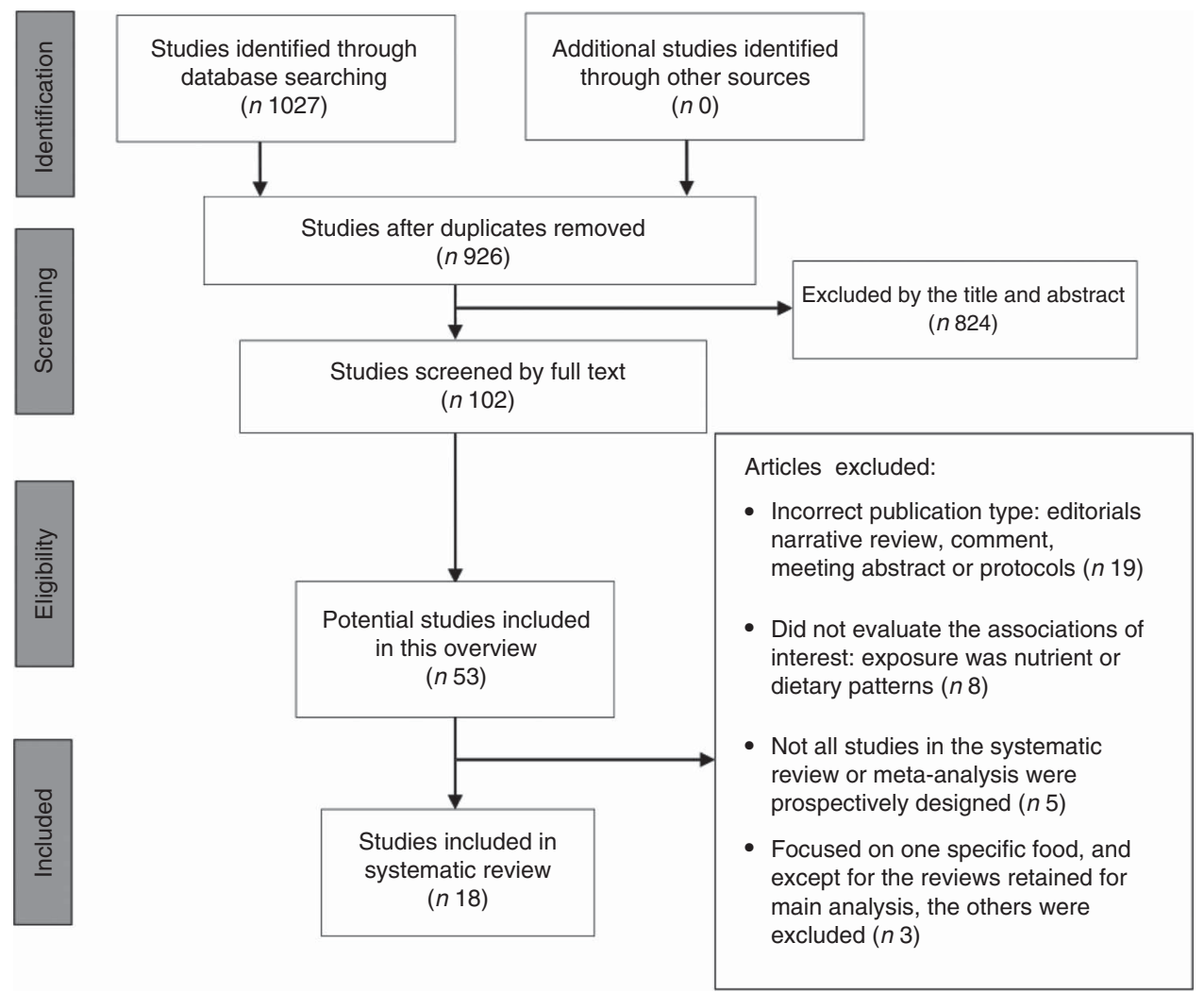

Fig. 1 Flow diagram of study selection for the present overview of systematic reviews and meta analyses on the reported associations of food groups with stroke risk

grains, whole grains, dairy products, eggs, chocolate, red and/or processed meat, fish, tea, sugar-sweetened beverages and coffee. Regarding the quality of the included reviews, eight were at the 'very good' level, six reviews were at the 'good' level, and four of the reviews were at the 'acceptable' level (Table 2).

\section{Nuts}

Two reviews reported on the relationship between nuts and risk of stroke or stroke mortality ${ }^{(6,12)}$. The method and conclusion of Shi et al.'s ${ }^{(12)}$ review were similar to those of Zhang et al. ${ }^{(6)}$; however, because the number of participants included was less than that of Zhang et al.'s, we only reported the results of the Zhang et al. review.

Zhang et $a l^{(6)}$ searched PubMed and EMBASE and reviewed the references of the retrieved articles. Nine prospective studies published from 2000 to 2013 and consisting of 476181 participants were included in their meta-analysis. Comparing the highest with the lowest nut consumption, the highest nut consumption was associated with a $10 \%$ lower risk of stroke (relative risk $(\mathrm{RR})=0.90 ; 95 \% \mathrm{CI} 0.83,0.98$ ). Gender modified the effects of nut consumption with a significant inverse association observed for women (RR= 0.88; 95\% CI 0.78, 0.98). Furthermore, although it did not reach significance, they observed a trend towards an inverse association between higher nut consumption and stroke mortality $(\mathrm{RR}=0.86 ; 95 \% \mathrm{CI} 0.69,1.06)$.

\section{Legumes}

One review reported on the relationship between legumes and stroke risk. Shi et al. ${ }^{(12)}$ searched MEDLINE and EMBASE up to January 2014. Six prospective studies published between 2000 and 2012 that included 173229 participants and 4030 stroke events were identified. The pooled results showed that a diet containing greater amounts of legumes might not be associated with a lower risk of stroke. Comparing the highest $v$. the lowest level of dietary legume intake, the pooled RR of stroke was 0.95 (95\% CI 0.84, 1.08).

\section{Fruits and vegetables}

Three reviews reported on the relationship between fruits and vegetables and stroke risk ${ }^{(3,13,14)}$. Among these three reviews, because $\mathrm{Hu}$ et al.'s ${ }^{(3)}$ review had the largest number of component studies with data on individual studies' effect sizes, we reported their results only. Hu et al. searched the PubMed (1947 to January 2014) and EMBASE (1974 to January 2014) databases. Twenty prospective studies, consisting of 760629 participants with 16981 stroke events, were included in that meta-analysis. Comparing the highest with the lowest category of total fruit and vegetable consumption, the highest category of total fruit and vegetable consumption was associated with a $21 \%$ lower risk of stroke ( $\mathrm{RR}=0.79 ; 95 \% \mathrm{CI} 0 \cdot 75,0 \cdot 84)$; moreover, fruit consumption was associated with a $23 \%$ 
Table 1 Characteristics of the systematic reviews on the reported associations of food groups with stroke risk included in the present overview

\begin{tabular}{|c|c|c|c|c|c|c|}
\hline Review & Targeted food & Outcome & $\begin{array}{l}\text { No. of } \\
\text { studies }\end{array}$ & $\begin{array}{l}\text { No. of } \\
\text { cases }\end{array}$ & $\begin{array}{l}\text { No. of } \\
\text { participants }\end{array}$ & Conclusion \\
\hline Zhang et al. (2015) $)^{(23)}$ & Tea & $\begin{array}{l}\text { Risk of stroke } \\
\text { Stroke } \\
\quad \text { mortality }\end{array}$ & $\begin{array}{l}8 \\
5\end{array}$ & $\begin{array}{c}10572 \\
2350\end{array}$ & 856206 & $\begin{array}{l}\text { Increased tea consumption is associated with a reduction in the risk of } \\
\text { stroke, cerebral infarction and intracerebral haemorrhage but has no } \\
\text { significant effect on stroke mortality or the risk of subarachnoid } \\
\text { haemorrhage }\end{array}$ \\
\hline Zhang et al. $(2015)^{(6)}$ & Nuts & $\begin{array}{l}\text { Risk of stroke } \\
\text { Stroke } \\
\quad \text { mortality }\end{array}$ & $\begin{array}{l}9 \\
2\end{array}$ & NR & 476181 & Nut consumption is inversely associated with risk of stroke \\
\hline Xi et al. $(2015)^{(25)}$ & SSB & Risk of stroke & 4 & 10011 & 259176 & $\begin{array}{l}\text { There was no significant association between SSB consumption and total } \\
\text { stroke }\end{array}$ \\
\hline Wu et al. (2015) $)^{(15)}$ & Refined grains & Risk of stroke & 8 & 8284 & 410821 & $\begin{array}{l}\text { A diet containing greater amounts of refined grains was not associated with } \\
\text { risk of stroke and its subtypes }\end{array}$ \\
\hline Qin et al. (2015) ${ }^{(17)}$ & Dairy products & Risk of stroke & 12 & 21801 & 504803 & $\begin{array}{l}\text { An inverse association was found between dairy consumption and stroke. } \\
\text { Low-fat dairy products and cheese may protect against stroke }\end{array}$ \\
\hline Shi et al. $(2014)^{(12)}$ & Legumes & Risk of stroke & 4 & 4030 & 173229 & Dietary legume intake was not associated with stroke risk \\
\hline Hu et al. $(2014)^{(3)}$ & Fruits and vegetables & Risk of stroke & 20 & 16981 & 760629 & $\begin{array}{l}\text { Fruit and vegetable consumption are inversely associated with the risk of } \\
\text { stroke }\end{array}$ \\
\hline Hu et al. (2014) ${ }^{(8)}$ & Dairy foods & Risk of CVD & 18 & 28318 & 764635 & Dairy foods might be inversely associated with the risk of stroke \\
\hline Ding et al. $(2014)^{(26)}$ & Coffee & Risk of CVD & 17 & 2030 & 1283685 & $\begin{array}{l}\text { A non-linear association between coffee consumption and stroke risk was } \\
\text { observed in meta-analysis }\end{array}$ \\
\hline Rong et al. (2013) ${ }^{(18)}$ & Eggs & Risk of stroke & 6 & 7579 & 210404 & $\begin{array}{l}\text { Higher consumption of eggs (up to } 1 \mathrm{egg} / \mathrm{d} \text { ) is not associated with increased } \\
\text { risk of stroke }\end{array}$ \\
\hline Chen et al. $(2013)^{(20)}$ & Red and processed meat & Risk of stroke & 5 & 9593 & 239251 & $\begin{array}{l}\text { Consumption of red and/or processed meat increased risk of stroke, } \\
\text { particularly ischaemic stroke }\end{array}$ \\
\hline Zhang et al. (2012) ${ }^{(7)}$ & Coffee & Risk of stroke & 13 & 12414 & 492760 & Coffee consumption at different levels reduces the risk of stroke \\
\hline Xun et al. (2012) $)^{(5)}$ & Fish & Risk of stroke & 16 & 10568 & 407127 & $\begin{array}{l}\text { Fish intake may have a protective effect against the risk of stroke, } \\
\text { particularly ischaemic stroke }\end{array}$ \\
\hline Shen et al. $(2012)^{(24)}$ & Tea & Risk of stroke & 14 & 10192 & 513804 & $\begin{array}{l}\text { Tea consumption is associated with a decreased risk of stroke, particularly } \\
\text { ischaemic stroke }\end{array}$ \\
\hline Larsson et al. (2012) ${ }^{(19)}$ & Chocolate & Risk of stroke & 5 & 4260 & 131345 & Moderate chocolate consumption may lower the risk of stroke \\
\hline Kaluza et al. $(2012)^{(4)}$ & Red meat consumption & Risk of stroke & 6 & 10630 & 329495 & $\begin{array}{l}\text { Consumption of fresh red meat and processed red meat as well as total red } \\
\text { meat is associated with increased risk of total stroke and ischaemic } \\
\text { stroke, but not haemorrhagic stroke }\end{array}$ \\
\hline $\begin{array}{l}\text { Larsson and Orsini } \\
\qquad(2011)^{(27)}\end{array}$ & Coffee & Risk of stroke & 11 & 10003 & 479689 & $\begin{array}{l}\text { Moderate coffee consumption may be weakly inversely associated with risk } \\
\text { of stroke }\end{array}$ \\
\hline Mellen et al. $(2008)^{(16)}$ & Whole grains & Risk of stroke & 4 & NR & NR & Greater whole grain intake was not associated with incident stroke \\
\hline
\end{tabular}

SSB, sugar-sweetened beverage; NR, not reported. 
Table 2 Quality of the systematic reviews on the reported associations of food groups with stroke risk included in the present overview

\begin{tabular}{|c|c|c|c|c|c|c|c|c|c|c|c|c|}
\hline \multirow[b]{2}{*}{ Review } & \multicolumn{11}{|c|}{ AMSTAR criterion } & \multirow[b]{2}{*}{ Total (yes) } \\
\hline & 1 & 2 & 3 & 4 & 5 & 6 & 7 & 8 & 9 & 10 & 11 & \\
\hline Zhang et al. ${ }^{(23)}$ & $Y$ & $\mathrm{Y}$ & $Y$ & $Y$ & $\mathrm{~N}$ & $Y$ & $Y$ & $Y$ & $Y$ & $\mathrm{Y}$ & $Y$ & 10 \\
\hline Zhang et al. ${ }^{(6)}$ & $\mathrm{Y}$ & Y & $Y$ & $\mathrm{~N}$ & $\mathrm{~N}$ & Y & Y & $Y$ & $\mathrm{Y}$ & $Y$ & Y & 9 \\
\hline Xi et al. & $\mathrm{Y}$ & $Y$ & $Y$ & $\mathrm{C}$ & $\mathrm{N}$ & $\mathrm{Y}$ & $\mathrm{Y}$ & $\mathrm{Y}$ & $\mathrm{Y}$ & $\mathrm{Y}$ & $\mathrm{Y}$ & 9 \\
\hline Wu et al. ${ }^{(15)}$ & $\mathrm{Y}$ & $Y$ & $\mathrm{Y}$ & $\mathrm{N}$ & $\mathrm{N}$ & $\mathrm{Y}$ & $\mathrm{Y}$ & $\mathrm{Y}$ & $\mathrm{Y}$ & $\mathrm{Y}$ & $\mathrm{N}$ & 8 \\
\hline Qin et al. ${ }^{(17)}$ & $\mathrm{Y}$ & $Y$ & $\mathrm{Y}$ & $\mathrm{C}$ & $\mathrm{N}$ & $\mathrm{Y}$ & $\mathrm{Y}$ & $\mathrm{Y}$ & $\mathrm{Y}$ & $\mathrm{Y}$ & $Y$ & 9 \\
\hline Shi et al..$^{(12)}$ & $Y$ & $Y$ & $\mathrm{Y}$ & $\mathrm{C}$ & $\mathrm{N}$ & $\mathrm{Y}$ & $\mathrm{Y}$ & $\mathrm{Y}$ & $\mathrm{Y}$ & $\mathrm{Y}$ & Y & 9 \\
\hline Hu et al. ${ }^{(3)}$ & $\mathrm{N}$ & C & $Y$ & $\mathrm{C}$ & $\mathrm{N}$ & $Y$ & $\mathrm{Y}$ & $\mathrm{Y}$ & $\mathrm{Y}$ & $\mathrm{Y}$ & $\mathrm{N}$ & 6 \\
\hline Hu et al. ${ }^{(8)}$ & Y & $\mathrm{Y}$ & $Y$ & $\mathrm{C}$ & $\mathrm{Y}$ & $Y$ & $Y$ & $\mathrm{Y}$ & $Y$ & $\mathrm{~N}$ & $Y$ & 9 \\
\hline Ding et al. ${ }^{(26)}$ & $\mathrm{N}$ & $Y$ & $\mathrm{~N}$ & $\mathrm{~N}$ & $\mathrm{Y}$ & $\mathrm{Y}$ & $Y$ & $Y$ & $\mathrm{Y}$ & $\mathrm{Y}$ & $Y$ & 8 \\
\hline Rong et al. (18) & Y & $Y$ & $Y$ & $\mathrm{~N}$ & $\mathrm{~N}$ & $Y$ & $Y$ & Y & $Y$ & Y & $Y$ & 9 \\
\hline Chen et al. ${ }^{(20)}$ & Y & Y & $\mathrm{N}$ & $\mathrm{N}$ & $\mathrm{N}$ & Y & $\mathrm{N}$ & Y & Y & Y & Y & 7 \\
\hline Zhang et al. ${ }^{(7)}$ & $Y$ & $Y$ & $Y$ & $Y$ & $\mathrm{~N}$ & $Y$ & $Y$ & $\mathrm{Y}$ & $\mathrm{Y}$ & $\mathrm{Y}$ & $\mathrm{N}$ & 9 \\
\hline Xun et al. ${ }^{(5)}$ & Y & $Y$ & $Y$ & C & $\mathrm{N}$ & Y & $\mathrm{N}$ & $\mathrm{Y}$ & Y & Y & Y & 8 \\
\hline Shen et al. & $Y$ & Y & $\mathrm{N}$ & $\mathrm{C}$ & $\mathrm{N}$ & Y & $\mathrm{N}$ & $\mathrm{N}$ & Y & Y & $\mathrm{N}$ & 5 \\
\hline Larsson et al. ${ }^{(19)}$ & NA & C & Y & $\mathrm{N}$ & $\mathrm{N}$ & Y & $\mathrm{N}$ & Y & Y & $\mathrm{N}$ & Y & 5 \\
\hline Kaluza et al. ${ }^{(4)}$ & $\mathrm{Y}$ & $\mathrm{Y}$ & $\mathrm{N}$ & C & $\mathrm{N}$ & Y & $\mathrm{N}$ & Y & Y & Y & Y & 7 \\
\hline Larsson and Orsini ${ }^{(27)}$ & NA & $Y$ & Y & $\mathrm{Y}$ & $\mathrm{N}$ & $\mathrm{Y}$ & $\mathrm{N}$ & $\mathrm{Y}$ & $Y$ & $\mathrm{Y}$ & $\mathrm{Y}$ & 8 \\
\hline Mellen et al. ${ }^{(16)}$ & NA & $\mathrm{Y}$ & $\mathrm{N}$ & $\mathrm{N}$ & $\mathrm{N}$ & $Y$ & $\mathrm{~N}$ & $\mathrm{Y}$ & $Y$ & $\mathrm{~N}$ & $Y$ & 5 \\
\hline
\end{tabular}

AMSTAR, Assessment of Multiple Systematic Reviews; Y, yes; N, no, NA, not applicable; C, can't answer.

lower risk of stroke $(\mathrm{RR}=0.77 ; 95 \% \mathrm{CI} 0.71,0.84)$ and vegetable consumption was associated with a $14 \%$ lower risk of stroke $(\mathrm{RR}=0.86 ; 95 \% \mathrm{CI} 0.79,0.93)$. The linear dose-response relationship showed that the risk of stroke decreased by $32 \%(\mathrm{RR}=0.68 ; 95 \%$ CI $0.56,0.82)$ and $11 \%$ $(\mathrm{RR}=0.89 ; 95 \% \mathrm{CI} 0.81,0.98)$ for every $200 \mathrm{~g} / \mathrm{d}$ increment in fruit consumption and vegetable consumption, respectively.

\section{Refined grains}

One review reported on the relationship between refined grains and risk of stroke. Wu et al. ${ }^{(15)}$ searched the MEDLINE (from 1 January 1966) and EMBASE (from 1 January 1974) databases up to 30 November 2014. Eight prospective studies consisting of 410821 subjects with 8284 stroke cases were included in the meta-analysis. The pooled results showed that a diet containing greater amounts of refined grains was not associated with risk of stroke $(\mathrm{RR}=1.02 ; 95 \% \mathrm{CI} 0.93,1.10)$. In the subgroup analysis, no significant associations between the consumption of refined grains and risk of stroke were found for both women and men, for both haemorrhagic and ischaemic strokes, and for both incident and fatal strokes. Consumption of white rice was not associated with risk of stroke (RR $=1 \cdot 01 ; 95 \%$ CI 0.93, 1.11). For those consuming 3 servings rice/d, the pooled results showed no significant association between refined grain consumption and total stroke $(\mathrm{RR}=0.98 ; 95 \%$ CI $0.73,1.03)$.

\section{Whole grains}

One review reported on the relationship between whole grains and risk of CVD. The results for stroke were extracted. Mellen et al. ${ }^{(16)}$ searched MEDLINE from 1966 to April 2006. In the analysis of four studies, greater whole grain intake ( 2.5 servings/d $v .0 .2$ servings/d) was not associated with stroke risk $(\mathrm{RR}=0.83 ; 95 \% \mathrm{CI} 0 \cdot 69,1 \cdot 02)$.

\section{Dairy products}

Two reviews reported on the relationship between dairy products and risk of stroke ${ }^{(8,17)}$. Qin et al. ${ }^{(17)}$ investigated the association between dairy products and the risk of major cardiovascular events. The results from patients with stroke were extracted. They searched the PubMed, EMBASE and Cochrane Library databases up to February 2014. Twenty studies (twelve in stroke) published between 1997 and 2013 and consisting of 504803 participants with 21801 stroke cases were included in the meta-analysis. Dairy consumption reduced the risk of stroke by $13 \%(\mathrm{RR}=0.87 ; 95 \%$ CI $0 \cdot 77,0.99)$. In the subgroup analysis, stroke risk was reduced by the consumption of low-fat dairy $(\mathrm{RR}=0.93 ; 95 \% \mathrm{CI} 0.88,0.99)$ and cheese $(\mathrm{RR}=0.91 ; 95 \% \mathrm{CI} 0.84,0.98)$.

Hu et al. ${ }^{(8)}$ searched EMBASE (1950 to November 2013), Web of Knowledge (1950 to November 2013) and PubMed (1945 to November 2013). Eighteen separate results from fifteen prospective cohort studies consisting of 764635 participants with 28138 stroke events were included in their meta-analysis. Total dairy $(R R=0.88$; $95 \%$ CI $0.82,0.94)$, low-fat dairy $(\mathrm{RR}=0.91 ; 95 \% \mathrm{CI} 0.85$, $0 \cdot 97)$, fermented milk ( $R R=0.80 ; 95 \%$ CI $0.71,089)$ and cheese ( $R R=0.94,95 \%$ CI 0.89, 0.99) were significantly associated with reduced risk of stroke, whereas whole/ high-fat dairy, non-fermented milk, butter and cream were not significantly associated with risk of stroke. No significant association with either ischaemic or haemorrhagic stroke was observed due to limited data. A non-linear dose-response relationship between milk and risk of stroke was found, and the RR $(95 \% \mathrm{CI})$ of stroke was 0.88 $(0.86,0.91), 0.82(0.79,0.86), 0.83(0.79,0.86), 0.85(0.81$, $0.89), 0.86(0.82,0.91), 0.91(0.84,0.98)$ and $0.94(0.86$, 1.02) for 100, 200, 300, 400, 500, 600 and $700 \mathrm{ml} \mathrm{milk/d,}$ respectively (Fig. 2; extracted from the original review). 


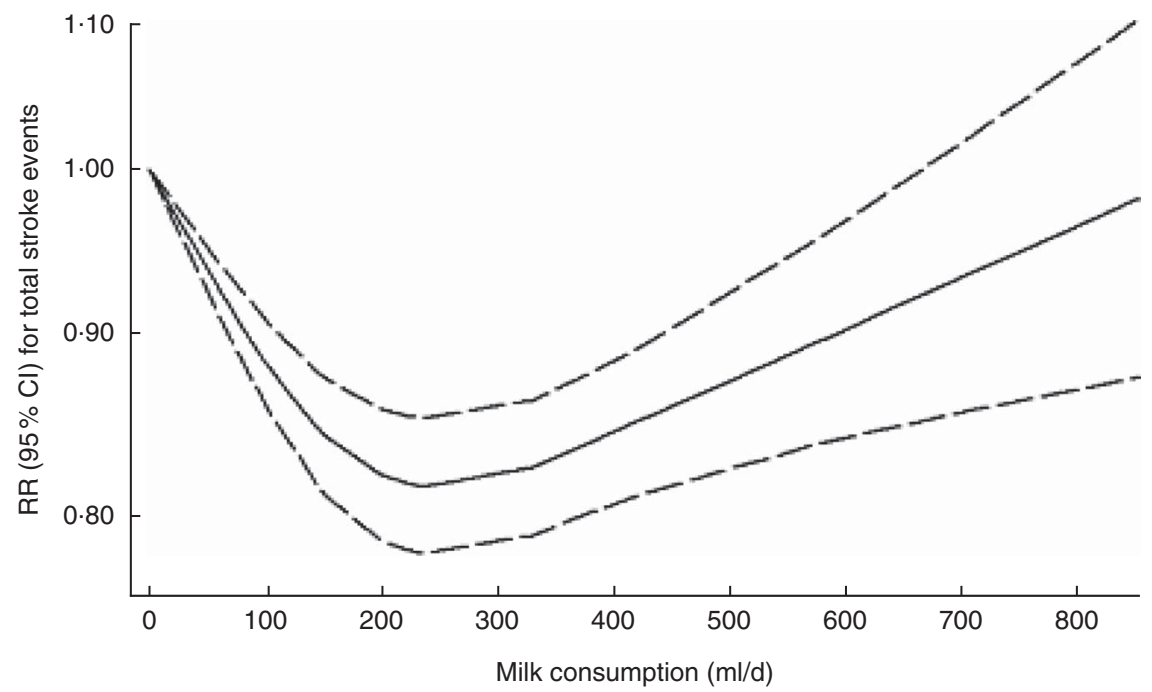

Fig. 2 Dose-response analysis between milk consumption and total stroke events; relative risk (RR; $95 \% \mathrm{Cl}(--\ldots)$. (From Hu et al. ${ }^{(8)}$ )

\section{Eggs}

One review reported on the relationship between eggs and risk of stroke. Rong et al. ${ }^{(18)}$ searched PubMed and EMBASE from January 1966 through June 2012. Six articles with eight reports consisting of 210404 patients with 7579 incident cases for stroke were included in their review. They did not observe a curvilinear association between egg consumption and risk of stroke. The pooled RR for increased consumption of $1 \mathrm{egg} / \mathrm{d}$ was 0.91 (95\% CI 0.81, 1.02). Higher intake of eggs (up to $1 \mathrm{egg} / \mathrm{d}$ ) was not associated with risk of stroke. However, those with higher egg consumption had a $25 \%$ lower risk of developing haemorrhagic stroke ( $R R=0.75 ; 95 \%$ CI $0.57,0.99)$.

\section{Chocolate}

One review reported on the relationship between chocolate and risk of stroke. Larsson et al. ${ }^{(19)}$ searched the PubMed and EMBASE databases through 13 January 2012. Five studies consisting of 131345 participants with 4260 stroke cases were included in their meta-analysis. Comparing the highest $v$. the lowest category of chocolate consumption, the highest chocolate consumption was associated with a $19 \%$ lower risk of stroke $(\mathrm{RR}=0 \cdot 81 ; 95 \%$ CI $0.73,0.90)$. A $50 \mathrm{~g} /$ week increment in chocolate consumption was associated with a $14 \%$ lower risk of stroke $(\mathrm{RR}=0 \cdot 86 ; 95 \%$ CI $0 \cdot 76,0 \cdot 97)$.

\section{Red and processed meat}

Two reviews reported on the relationship between red and processed meat and risk of stroke ${ }^{(4,20)}$. Chen et al. ${ }^{(20)}$ performed a literature search in the PubMed database through June 2012. Five studies consisting of 239251 subjects with 9593 stroke events were identified. Comparing the highest category of consumption with the lowest category, the highest categories of total meat (red and processed meat combined) consumption, red meat consumption and processed meat consumption were associated with a $15 \%(\mathrm{RR}=1 \cdot 15 ; 95 \% \mathrm{CI} 1 \cdot 05,1 \cdot 25), 9 \%$ $(\mathrm{RR}=1 \cdot 09 ; 95 \% \mathrm{CI} 1 \cdot 01,1 \cdot 18)$ and $14 \%(\mathrm{RR}=1 \cdot 14 ; 95 \% \mathrm{CI}$ $1.05,1.25)$ higher risk of total stroke, respectively. The pooled RR of ischaemic stroke (highest $v$. lowest quintile) was 1.15 (95\% CI 1.04, 1.28), 1.13 (95\% CI 1.01, 1.25) and $1 \cdot 19$ (95\% CI 1.08, 1.31), respectively. Consumption of red and/or processed meat was not associated with haemorrhagic stroke. In the dose-response analysis, the risk of stroke increased significantly by 10 and $13 \%$ for each $100 \mathrm{~g} / \mathrm{d}$ increment in total and red meat consumption, respectively, and by $11 \%$ for each $50 \mathrm{~g} / \mathrm{d}$ increment in processed meat consumption.

Kaluza et al. ${ }^{(4)}$ searched the PubMed database through 26 May 2012. Five articles including the results from six prospective studies published between 2003 and 2012 and consisting of 329495 participants with 10630 stroke cases were included in their meta-analysis. Increased consumption of 1 serving/d for fresh red meat, processed meat and total red meat was associated with $11 \%(\mathrm{RR}=$ $1 \cdot 11 ; 95 \%$ CI $1.03,1.20), 13 \%(\mathrm{RR}=1.1395 \%$ CI 1.03 , $1 \cdot 24)$ and $11 \%(\mathrm{RR}=1 \cdot 11 ; 95 \% \mathrm{CI} 1 \cdot 06,1 \cdot 16)$ higher risk of total stroke, respectively. In the subgroup analysis, the risk of ischaemic stroke was positively associated with the consumption of fresh red meat $(\mathrm{RR}=1 \cdot 13 ; 95 \%$ CI $1 \cdot 00$, $1 \cdot 27)$, processed meat $(\mathrm{RR}=1 \cdot 15 ; 95 \% \mathrm{CI} 1 \cdot 06,1 \cdot 24)$ and total red meat $(\mathrm{RR}=1 \cdot 12 ; 95 \% \mathrm{CI} 1 \cdot 05,1 \cdot 19)$; no significant associations were observed for haemorrhagic stroke.

\section{Fish}

Three reviews reported on the relationship between fish and risk of stroke ${ }^{(5,21,22)}$. Among these three reviews, Xun et al.'s ${ }^{(5)}$ had the largest number of component studies with data on the individual studies' effect sizes; thus, we reported its results only. They searched MEDLINE and EMBASE through April 2012 and reviewed the reference 
lists of the relevant articles or retrieved additional information through Google. Sixteen eligible studies (nineteen cohorts), consisting of 402127 individuals with 10568 incident cases, were included in their meta-analysis. Compared with those who never consumed fish or those who ate $<1$ fish serving/month, those who consumed 1 fish serving/week, 2-4 fish servings/week and $\geq 5$ fish servings/week had a 14\% (hazard ratio $(\mathrm{HR})=0 \cdot 86 ; 95 \%$ CI $0.80,0.93), 9 \%(\mathrm{HR}=0.91 ; 95 \%$ CI $0.85,0.98)$ and $13 \%$ (HR $=0.87 ; 95 \%$ CI 0.79, 0.96), respectively, lower risk of stroke. In the subgroup analysis, an inverse association between fish intake and stroke incidence was found only by those studies conducted in North America.

\section{Tea}

Two reviewers reported on the relationship between tea consumption and risk of stroke or stroke mortality ${ }^{(23,24)}$. Zhang et $a l^{(23)}$ investigated the association between tea consumption and the risk of major CVD. The results from patients with stroke were extracted. They searched the PubMed, EMBASE and Cochrane Library electronic databases up to July 2014. Twenty-four studies (stroke in eight studies, stroke mortality in five studies) with 10572 cases of stroke and 2350 stroke deaths among 856206 individuals were included in their meta-analysis. According to high $v$. low tea consumption, high tea consumption was associated with a $23 \%$ lower risk of stroke $(\mathrm{RR}=0.67 ; 95 \% \mathrm{CI} 0.53,0.85)$ and a $22 \%$ lower risk of cerebral infarction $(\mathrm{RR}=0.78$; $95 \% \mathrm{CI} 0.67$, 0.92 ), but not with any reduction in stroke mortality, intracerebral haemorrhage or subarachnoid haemorrhage. The dose-response analysis indicated that an increase in tea consumption by 3 cups/d was associated with an $18 \%$ lower risk of stroke ( $\mathrm{RR}=0.82 ; 95 \% \mathrm{CI} 0 \cdot 73,0.92)$, a $16 \%$ lower risk of cerebral infarction (RR $=0.84 ; 95 \%$ CI $0.72,0.98)$ and an $11 \%$ lower risk of intracerebral haemorrhage ( $\mathrm{RR}=0.79 ; 95 \%$ CI $0.72,0 \cdot 87$ ), albeit with little or no effect on stroke mortality $(\mathrm{RR}=0.93 ; 95 \% \mathrm{CI} 0.83,1.05)$. In the subgroup analysis, they found that an increase of 3 cups tea/d was associated with a reduced risk of stroke if the participants were men, Asian, or if they consumed green tea.

Shen et al. ${ }^{(24)}$ searched the PubMed database from January 1966 to March 2012 and reviewed the reference lists of the retrieved articles to identify relevant studies. Fourteen studies consisting of 513804 participants with 10192 stroke cases were included in their meta-analysis. An increase of $3 \mathrm{cups} / \mathrm{d}$ in tea consumption was associated with a $13 \%$ lower risk of stroke $(\mathrm{RR}=0 \cdot 87 ; 95 \% \mathrm{CI}$ $0 \cdot 81,0.94)$. Tea consumption was also associated with a $24 \%$ lower risk of ischaemic stroke $(\mathrm{RR}=0.76$; $95 \%$ CI $0.69,0.84)$ but not with cerebral haemorrhage or subarachnoid haemorrhage.

\section{Sugar-sweetened beverages}

One review reported on the relationship between sugarsweetened beverages (SSB) and risk of stroke. Xi et al. ${ }^{(25)}$ investigated the relationship between SSB and risk of hypertension and CVD. The results from patients with stroke were extracted. They searched the PubMed and EMBASE databases up to 5 May 2014. Four prospective studies consisting of 259176 participants with 10011 incident cases of stroke were included in their metaanalysis. For every 1 serving/d increase in SSB consumption, the pooled results showed that no significant association existed between SSB consumption and total stroke ( $\mathrm{RR}=1.06$; $95 \% \mathrm{CI} 0.97,1.15)$ Compared with the lowest level, the highest intake level of SSB was marginally associated with the overall risk of stroke $(\mathrm{RR}=1 \cdot 10 ; 95 \%$ CI 1.00, 1.20). In the subgroup analysis, they found no significant associations between SSB intake and the risk of either ischaemic stroke or haemorrhagic stroke. However, SSB intake was associated with a higher risk of stroke in Caucasians ( $\mathrm{RR}=1 \cdot 17$; $95 \% \mathrm{CI} 1 \cdot 06,1 \cdot 28)$, although not in East Asians.

\section{Coffee}

Three reviews reported on the relationship between coffee and stroke $\mathrm{f}^{(7,26,27)}$. Ding et al. $^{(26)}$ performed a metaanalysis to assess the dose-response relationship between long-term coffee consumption and risk of CVD. The results from patients with stroke were extracted. They searched the PubMed and EMBASE databases from January 1966 to March 2013. Thirty-six studies (seventeen studies on stroke) consisting of 1283685 study participants with 2030 stroke cases were included. The categories of coffee consumption in their review were defined as follows: the lowest category (median and mean coffee consumption $=0 \mathrm{cups} / \mathrm{d})$, the third highest $($ median $=1.5$ cups/d; mean $=1.48 \mathrm{cups} / \mathrm{d}$ ), the second highest (med$\operatorname{ian}=3.5 \mathrm{cups} / \mathrm{d}$; mean $=3 \mathrm{cups} / \mathrm{d}$ ) and the highest category $($ median $=5 \mathrm{cups} / \mathrm{d}$; mean $=5.5 \mathrm{cups} / \mathrm{d}$ ). Compared with the lowest category of coffee consumption, the corresponding $\mathrm{RR}$ of stroke was 0.89 (95\% CI 0.84, 0.94) for the third highest category, $0.80(95 \% \mathrm{CI} 0 \cdot 75,0 \cdot 86)$ for the second highest category and 0.95 (95\% CI $0.84,1.07)$ for the highest category. Additionally, they found that coffee consumption was non-linearly associated with stroke risk (Fig. 3; extracted from the original article).

Zhang et $a l{ }^{(7)}$ searched PubMed and EMBASE up to June 2011. Thirteen studies consisting of 492760 individuals with 12414 stroke cases were included in their metaanalysis. They combined coffee consumption of less than 1 cup/week and no coffee consumption into the noncoffee consumption group. Coffee consumption of more than 1 cup/d was further classified into three categories: (i) light consumption: $>1 \mathrm{cup} /$ week but $\leq 1 \mathrm{cup} / \mathrm{d}$ in the US and Asian studies and $>1 \mathrm{cup} /$ week but $\leq 2 \mathrm{cups} / \mathrm{d}$ in the European studies; (ii) moderate consumption: 1-3 cups/d in the US and Asian studies and 3-4 cups/d in the European studies; and (iii) heavy consumption: $\geq 4 \mathrm{cups} / \mathrm{d}$ in the US and Asian studies and $\geq 5 \mathrm{cups} / \mathrm{d}$ in the European studies. Compared with non-coffee drinking, all coffee 


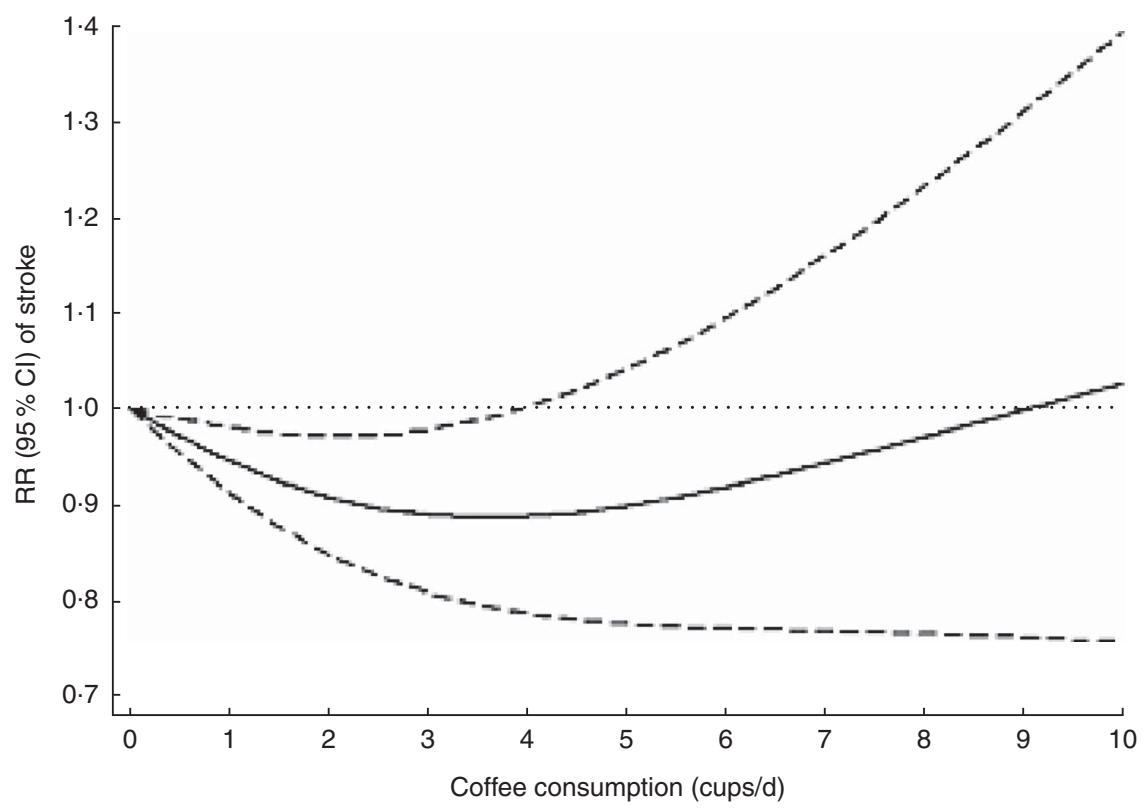

Fig. 3 Coffee consumption and risk of stroke; relative risk (RR; heterogeneity $=0.007, P$ for trend $<0.001, P$ for non-linearity $<0.001$. (From Ding et al. ${ }^{(26)}$ )

consumption reduced total stroke risk by $11 \%(\mathrm{RR}=0 \cdot 89$; 95\% CI 0.81, 0.97). Coffee consumption reduced stroke risk by $17 \%(\mathrm{RR}=0 \cdot 83 ; 95 \% \mathrm{CI} 0 \cdot 79,0 \cdot 87)$ among females. Although lacking significance for men, coffee consumption tended to decrease stroke risk $(\mathrm{RR}=0.88 ; 95 \% \mathrm{CI}$ $0.72,1.09)$. Light, moderate and heavy coffee consumption reduced all-type stroke risk by $10 \%(\mathrm{RR}=0 \cdot 90 ; 95 \% \mathrm{CI}$ $0.85,0.96), 14 \%(\mathrm{RR}=0.86 ; 95 \% \mathrm{CI} 0.76,0.97)$ and $17 \%$ $(\mathrm{RR}=0.83 ; 95 \% \mathrm{CI} 0 \cdot 78,0 \cdot 89)$, respectively.

Larsson and Orsini ${ }^{(27)}$ searched PubMed and EMBASE from January 1966 through May 2011. Eleven studies consisting of 479689 participants with 10003 stroke cases were included in their meta-analysis. In their review, coffee consumption was classified into five groups: reference (the lowest category in each study), <3 cups/d, 3-5 cups/ d, 5-7 cups/d and $>7 \mathrm{cups} / \mathrm{d}$. Compared with no coffee consumption, 2 cups coffee/d reduced stroke risk by $14 \%$ $(\mathrm{RR}=0 \cdot 86 ; 95 \%$ CI 0.78, 0.94), 3-4 cups/d reduced risk of stroke by $17 \%(\mathrm{RR}=0.83 ; 95 \% \mathrm{CI} 0.74,0.92)$ and $6 \mathrm{cups} / \mathrm{d}$ reduced risk of stroke by $13 \%(\mathrm{RR}=0 \cdot 87 ; 95 \% \mathrm{CI} 0 \cdot 77$, 0.97), with no significant difference observed between 8 cups/d and stroke risk. The results demonstrated that a non-linear association existed between coffee consumption and risk of stroke (Fig. 4; extracted from the original article). The findings from that meta-analysis indicated that moderate coffee consumption might be weakly inversely associated with stroke risk.

\section{Discussion}

We have provided an overview of systematic reviews and meta-analyses relating to a specific food or to food groups that have been associated with stroke outcome. Our overview synthesized the evidence from eighteen reviews. The quality of these systematic reviews or meta-analyses is generally good. Overall, thirteen specific foods were studied for an association with stroke outcome, including nuts, legumes, fruits and vegetables, refined grains, whole grains, dairy products, eggs, chocolate, red and/or processed meat, fish, tea, SSB and coffee. While high consumption of nuts, fruits, vegetables, dairy foods, fish and tea, and moderate consumption of coffee and chocolate demonstrated a protective effect, high consumption of red and/or processed meat was associated with increased stroke risk. Refined grain, SSB, legume, egg and whole grain intake showed no effect on stroke outcome.

There are some common characteristics and nutrition factors for protective foods, which are described as follows. Nuts contain mostly unsaturated fats, which have beneficial effects on blood lipids ${ }^{(28)}$. Moreover, nuts are rich in arginine. Arginine is the precursor of nitric oxide, which is an effective vasodilator and may inhibit platelet aggregation and adhesion. Additionally, nuts are rich sources of $\mathrm{K}, \mathrm{Mg}$, folate and fibre, and meta-analyses have shown that supplementation with $\mathrm{K}$ alone or in combination with other minerals such as $\mathrm{Mg}$ and $\mathrm{Ca}$ resulted in a reduction in blood pressure ${ }^{(29)}$. Folate is a crucial regulator of the metabolism of homocysteine, and studies have indicated that dietary supplementation with folate reduced blood levels of homocysteine and was inversely related to the risk of stroke ${ }^{(30)}$. Studies have also suggested that higher dietary fibre and protein intake might lower blood pressure and were associated with a reduced risk for stroke ${ }^{(31,32)}$.

Complex mechanisms might explain the protective effects of fruits and vegetables on stroke. Studies have 


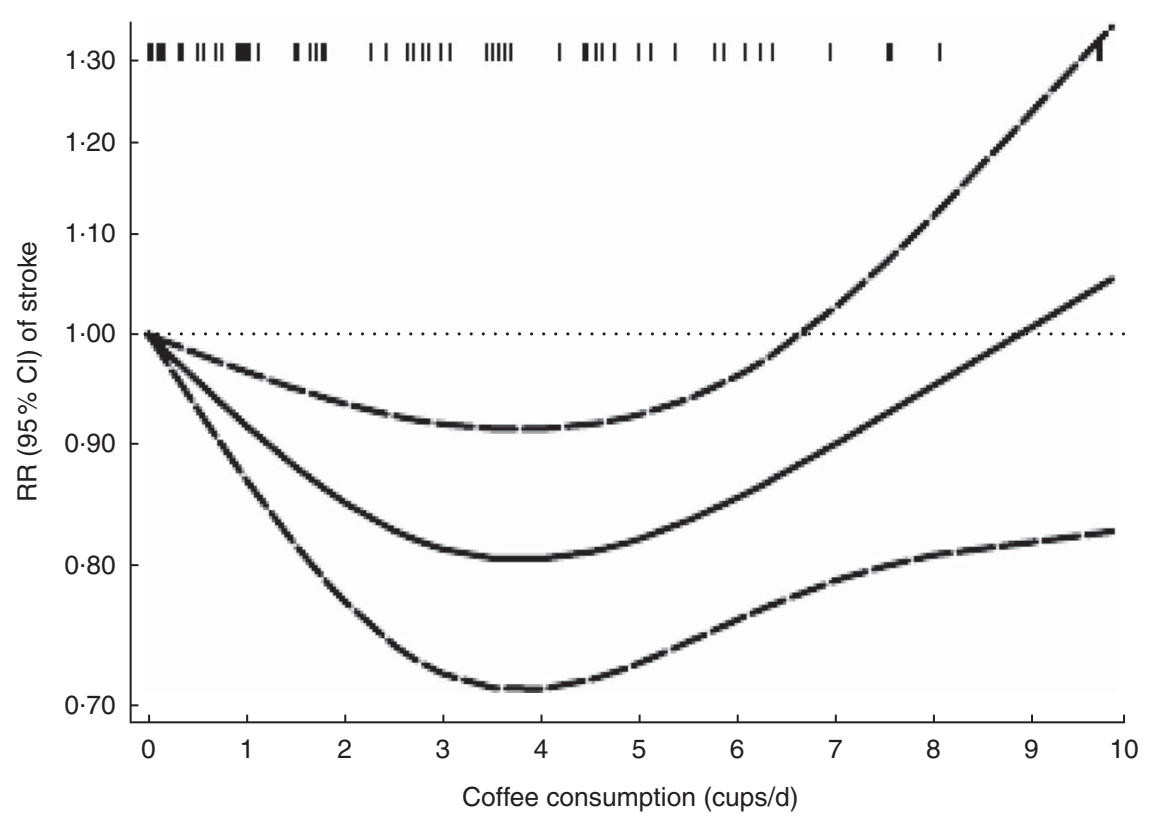

Fig. 4 Adjusted relative risk of stroke associated with coffee consumption in a meta-analysis of published studies, 1966-2011; relative risk $(\mathrm{RR} ;-)$ and $95 \% \mathrm{Cl}(-\ldots-\ldots) ; \ldots$ represents no effect. (From Larsson and Orsini ${ }^{(27)}$ )

shown that increased fruit and vegetable consumption might lower blood pressure, improve microvascular function and have favourable effects on other cardiovascular risk factors, such as those observed for BMI, waist circumference, total cholesterol, LDL cholesterol, inflammation and oxidative stress ${ }^{(3)}$. Fruits and vegetables are rich sources of $\mathrm{K}$, folate, antioxidants (e.g. vitamin $\mathrm{C}$, $\beta$-carotene, flavonoids) and fibre, which were significantly associated with decreased stroke risk ${ }^{(14)}$.

Several biological mechanisms may be involved in the potentially protective effects of dairy products against CVD. Dairy products are rich in minerals ( $\mathrm{Ca}, \mathrm{K}$ and $\mathrm{Mg}$ ), protein (casein and whey) and vitamins (riboflavin and vitamin $\mathrm{B}_{12}$ ). Such components showed beneficial effects on CVD incidence. However, the underlying mechanisms of the effects of dairy products on stroke remain unknown ${ }^{(8)}$.

The reason why fish has beneficial attributes in lowering the risk of stroke may be attributed to the antithrombotic activity and anti-inflammatory property of long-chain $n$-3 PUFA, the key nutrients in fish ${ }^{(33,34)}$. Such marine-derived long-chain $n$ - 3 PUFA have been shown to have multiple favourable effects on blood pressure, arrhythmias, lipid profile, platelet aggregation and endothelial function that might reduce the risk of ischaemic stroke ${ }^{(21)}$.

The beneficial effect of tea consumption on stroke risk has been suggested to be attributable to the presence of polyphenolic compounds, particularly catechins, in green and black tea. Catechins, a major category of polyphenols in tea, exert a wide spectrum of beneficial effects against CVD, including antioxidative, anti-inflammatory, anti-endothelial dysfunction, antihypertensive and lipid-lowering effects ${ }^{(24,35)}$.

Coffee is a complex mixture of biologically active substances that may have both beneficial and harmful effects on the cardiovascular system. The phenolic compounds in coffee, such as caffeic, ferulic and p-coumaric acids, have strong antioxidant activity and may reduce the oxidation of LDL cholesterol ${ }^{(27)}$. Additionally, habitual coffee consumption has been associated with higher insulin sensitivity, and several studies have found an inverse association between coffee consumption and blood concentrations of some inflammatory markers. Alternatively, caffeine in coffee may increase blood pressure and levels of plasma homocysteine. The non-linear U-shaped relationship between coffee consumption and risk of stroke might be due to a combination of beneficial and detrimental effects.

Chocolate is a source of flavonoids and is particularly rich in epicatechin, catechin and procyanidins (polymers of catechins and epicatechins). The flavonoids may reduce the risk of stroke through several biological mechanisms, including antioxidant, antiplatelet and anti-inflammatory effects, as well as by lowering blood pressure, increasing HDL cholesterol and improving endothelial function ${ }^{(36)}$. Chocolate also contains caffeine, and the consumption of coffee, which is a rich source of caffeine, has been inversely associated with risk of stroke ${ }^{(7)}$.

Foroughi et al. ${ }^{(37)}$ and Sherzai et al. ${ }^{(9)}$ summarized the evidence regarding the relationship between nutrition and stroke outcome. However, the type of studies included in their reviews was not limited. Mixed results were demonstrated. Compared with previous reviews of nutrition and stroke, our overview is robust because only systematic reviews and meta-analyses of prospective observational studies were included, which might eliminate selection and recall bias. In addition, the systematic reviews and metaanalyses included in our overview have relatively large sample sizes and long periods of follow-up. The findings 
from our study are informative because they provide a high-evidence overview of food groups associated with stroke outcome, which researchers and clinicians may use as a basis for strong practice recommendations.

Our overview has some limitations. Because all original studies in the systematic reviews or meta-analyses included in our overview followed an observational design, the possibility that other factors might account for the observed results could not be eliminated. Additionally, most original studies used FFQ to evaluate the levels of food consumption, and measurement error was inevitable. Furthermore, the cut-off values for the food consumption categories were not consistent among the studies. These limitations might have contributed to heterogeneity in the pooled analysis. One cannot conclude convincingly from those data that increasing consumption of some types of foods has a protective effect against stroke outcomes. Future studies should use uniform criteria for food consumption. Dietary patterns differed in different populations, and habitual consumption of foods also differed. For example, green tea consumption is more popular in Asian than in Western countries. However, analyses stratified by ethnicity were not available in most of the systematic reviews included in our overview, which might play an important role in the relationship between food consumption and stroke outcome. Finally, the findings from reviews receiving lower quality ratings and those of small size must be interpreted with caution.

\section{Conclusion}

The present study is the first overview of systematic reviews that brings together the evidence relating to food groups associated with stroke outcome. It shows that the quality of these systematic reviews is generally good. Overall, thirteen specific foods have been studied for an association with stroke outcome. Whereas a high consumption of nuts, fruits, vegetables, dairy foods, fish and tea, and moderate consumption of coffee and chocolate demonstrated a protective effect, a high consumption of red and/or processed meat was associated with increased stroke risk. Refined grain, SSB, legume, egg and whole grain intake showed no effect on stroke outcome. Although the current overview provides powerful evidence on food groups associated with stroke risk, its findings require further confirmation by welldesigned large randomized clinical trials.

\section{Acknowledgements}

Financial support: This research received no specific grant from any funding agency in the public, commercial or not-for-profit sectors. Conflict of interest: The authors declare that they have no conflicts of interest. Authorship: C.D. and Q.L. contributed equally to this work. C.D. and Q.L. designed the study; C.D. and Q.L. searched the databases, checked them according to the eligibility and exclusion criteria and analysed the data; B.G., L.L. and L.C. assisted C.D. and Q.L. in data collection and statistical analysis. C.D. wrote the draft of the paper; C.D., Q.L., B.G., L.L., L.C., Y.Z. and L.F. revised the paper. Y.Z. and L.F. were responsible for the final content. All the authors read and approved the final manuscript. Ethics of buman subject participation: Not applicable.

\section{Supplementary material}

To view supplementary material for this article, please visit https://doi.org/10.1017/\$1368980017003093

\section{References}

1. Yang G, Wang Y, Zeng Y et al. (2013) Rapid health transition in China, 1990-2010: findings from the Global Burden of Disease Study 2010. Lancet 381, 1987-2015.

2. Meschia JF, Bushnell C, Boden-Albala B et al. (2014) Guidelines for the primary prevention of stroke: a statement for healthcare professionals from the American Heart Association/American Stroke Association. Stroke 45, 3754-3832.

3. Hu D, Huang J, Wang Y et al. (2014) Fruits and vegetables consumption and risk of stroke: a meta-analysis of prospective cohort studies. Stroke 45, 1613-1619.

4. Kaluza J, Wolk A \& Larsson SC (2012) Red meat consumption and risk of stroke: a meta-analysis of prospective studies. Stroke 43, 2556-2560.

5. Xun P, Qin B, Song Y et al. (2012) Fish consumption and risk of stroke and its subtypes: accumulative evidence from a meta-analysis of prospective cohort studies. Eur J Clin Nutr 66, 1199-1207.

6. Zhang Z, Xu G, Wei Y et al. (2015) Nut consumption and risk of stroke. Eur J Epidemiol 30, 189-196.

7. Zhang R, Wang Y, Song B et al. (2012) Coffee consumption and risk of stroke: a meta-analysis of cohort studies. Cent Eur J Med 7, 310-316.

8. Hu D, Huang J, Wang Y et al. (2014) Dairy foods and risk of stroke: a meta-analysis of prospective cohort studies. Nutr Metab Cardiovasc Dis 24, 460-469.

9. Sherzai A, Heim LT, Boothby C et al. (2012) Stroke, food groups, and dietary patterns: a systematic review. Nutr Rev 70, $423-435$.

10. Moher D, Liberati A, Tetzlaff J et al. (2010) Preferred reporting items for systematic reviews and meta-analyses: the PRISMA statement. Int J Surg 8, 336-341.

11. Shea BJ, Hamel C, Wells GA et al. (2009) AMSTAR is a reliable and valid measurement tool to assess the methodological quality of systematic reviews. J Clin Epidemiol 62, 1013-1020.

12. Shi ZQ, Tang JJ, Wu H et al. (2014) Consumption of nuts and legumes and risk of stroke: a meta-analysis of prospective cohort studies. Nutr Metab Cardiovasc Dis 24, 1262-1271.

13. He FJ, Nowson CA \& MacGregor GA (2006) Fruit and vegetable consumption and stroke: meta-analysis of cohort studies. Lancet 367, 320-326.

14. Dauchet L, Amouyel P \& Dallongeville J (2005) Fruit and vegetable consumption and risk of stroke: a meta-analysis of cohort studies. Neurology 65, 1193-1197.

15. Wu D, Guan Y, Lv S et al. (2015) No evidence of increased risk of stroke with consumption of refined grains: a metaanalysis of prospective cohort studies. J Stroke Cerebrovasc Dis 24, 2738-2746. 
16. Mellen PB, Walsh TF \& Herrington DM (2008) Whole grain intake and cardiovascular disease: a meta-analysis. Nutr Metab Cardiovasc Dis 18, 283-290.

17. Qin LQ, Xu JY, Han SF et al. (2015) Dairy consumption and risk of cardiovascular disease: an updated meta-analysis of prospective cohort studies. Asia Pac J Clin Nutr 24, 90-100.

18. Rong Y, Chen L, Zhu T et al. (2013) Egg consumption and risk of coronary heart disease and stroke: dose-response meta-analysis of prospective cohort studies. BMJ346, e8539.

19. Larsson SC, Virtamo J \& Wolk A (2012) Chocolate consumption and risk of stroke: a prospective cohort of men and meta-analysis. Neurology 79, 1223-1229.

20. Chen GC, Lv DB, Pang Z et al. (2013) Red and processed meat consumption and risk of stroke: a meta-analysis of prospective cohort studies. Eur J Clin Nutr 67, 91-95.

21. He K, Song Y, Daviglus ML et al. (2004) Fish consumption and incidence of stroke: a meta-analysis of cohort studies. Stroke 35, 1538-1542.

22. Larsson SC \& Orsini N (2011) Fish consumption and the risk of stroke: a dose-response meta-analysis. Stroke 42, 3621-3623.

23. Zhang C, Qin YY, Wei X et al. (2015) Tea consumption and risk of cardiovascular outcomes and total mortality: a systematic review and meta-analysis of prospective observational studies. Eur J Epidemiol 30, 103-113.

24. Shen L, Song LG, Ma H et al. (2012) Tea consumption and risk of stroke: a dose-response meta-analysis of prospective studies. J Zhejiang Univ Sci B 13, 652-662.

25. Xi B, Huang Y, Reilly KH et al. (2015) Sugar-sweetened beverages and risk of hypertension and CVD: a doseresponse meta-analysis. Br J Nutr 113, 709-717.

26. Ding M, Bhupathiraju SN, Satija A et al. (2014) Long-term coffee consumption and risk of cardiovascular disease: a systematic review and a dose-response meta-analysis of prospective cohort studies. Circulation 129, 643-659.
27. Larsson SC \& Orsini N (2011) Coffee consumption and risk of stroke: a dose-response meta-analysis of prospective studies. Am J Epidemiol 174, 993-1001.

28. Grundy SM \& Denke MA (1990) Dietary influences on serum lipids and lipoproteins. J Lipid Res 31, 1149-1172.

29. Beyer FR, Dickinson HO, Nicolson DJ et al. (2006) Combined calcium, magnesium and potassium supplementation for the management of primary hypertension in adults. Cochrane Database Syst Rev issue 3, CD004805.

30. Wang X, Qin X, Demirtas H et al. (2007) Efficacy of folic acid supplementation in stroke prevention: a meta-analysis. Lancet 369, 1876-1882.

31. Streppel MT, Arends LR, van 't Veer P et al. (2005) Dietary fiber and blood pressure: a meta-analysis of randomized placebo-controlled trials. Arch Intern Med 165, 150-156.

32. Zhang Z, Xu G, Liu D et al. (2013) Dietary fiber consumption and risk of stroke. Eur J Epidemiol 28, 119-130.

33. He K (2009) Fish, long-chain omega-3 polyunsaturated fatty acids and prevention of cardiovascular disease - eat fish or take fish oil supplement? Prog Cardiovasc Dis 52, 95-114.

34. Calder PC (2004) n-3 Fatty acids and cardiovascular disease: evidence explained and mechanisms explored. Clin Sci (Lond) 107, 1-11.

35. Babu PV \& Liu D (2008) Green tea catechins and cardiovascular health: an update. Curr Med Chem 15, 1840-1850.

36. Natsume M, Osakabe N, Yamagishi M et al. (2000) Analyses of polyphenols in cacao liquor, cocoa, and chocolate by normal-phase and reversed-phase HPLC. Biosci Biotechnol Biochem 64, 2581-2587.

37. Foroughi M, Akhavanzanjani M, Maghsoudi Z et al. (2013) Stroke and nutrition: a review of studies. Int J Prev Med $\mathbf{4}$, Suppl. 2, S165-S179. 